\title{
In vitro study on human cytomegalovirus affecting early pregnancy villous EVT's invasion function
}

Liu Tao ${ }^{1,2}$, Chen Suhua ${ }^{1 *}$, Chen Juanjuan', Yin Zongzhi', Xiao Juan, Zhang Dandan

\begin{abstract}
Background: Human cytomegalovirus (HCMV) is the most common pathogen in uterus during pregnancy, which may lead to some serious results such as miscarriage, stillbirth, cerebellar malformation, fetus developmental retardation, but its pathogenesis has not been fully explained. The hypofunction of extravillous cytotrophoblast (EVT) invasion is the essential pathologic base of some complications of pregnancy. c-erbB-2 is a kind of oncogene protein and closely linked with embryogenesis, tissue repair and regeneration. Matrix metalloproteinase (MMP) is one of the key enzymes which affect EVT migration and invasion function. The expression level changes of c-erbB2, MMP-2 and MMP-9 can reflect the changes of EVT invasion function.
\end{abstract}

Results: To explore the influence of HCMV on the invasion function of EVT, we tested the protein expression level changes of c-erbB-2, MMP-2 and MMP-9 in villous explant cultured in vitro infected by HCMV, with the use of immunohistochemistry SP method and western blot. We confirmed that HCMV can reproduce and spread in early pregnancy villus; c-erbB-2 protein mainly expressed in normal early pregnancy villous syncytiotrophoblast (ST) remote plasma membrane and EVT, especially remote EVT cell membrane in villous stem cell column, little expressed in ST proximal end cell membrane and interstitial cells; MMP-2 protein primarily expressed in early pregnancy villous EVT endochylema and rarely in villous trophoblast (VT), ST and interstitial cells; MMP-9 protein largely expressed in early pregnancy villous mesenchyme, EVT and VT endochylema. Compared with control group, the three kinds of protein expression level in early pregnancy villus of virus group significantly decreased $(P<0.05)$.

Conclusion: HCMV can infect villus in vitro and cause the decrease of early pregnancy villous EVT's invasion function.

\section{Introduction}

Human cytomegalovirus (HCMV) is the most common pathogen in uterus during pregnancy, which may lead to some serious results such as miscarriage, stillbirth, cerebellar malformation, fetus developmental retardation, but its pathogenesis has not been fully explained [1]. It is currently considered that in later stage of blastula's nidation, cytotrophoblast (CT) differentiates to villous trophoblast (VT) and extravillous cytotrophoblast (EVT). VT is active in karyokinesis, fusing to form syncytiotrophoblast (ST). ST performs the functions of internal secretion, immunity and material exchange and so on; EVT forms cell column after its proliferation and migration, invasively grow towards uterus mesenchyme

\footnotetext{
* Correspondence: Chen_Suhua@mailhust.edu.cn

'Department of Obsterics and Gynecology, Tongji Hospital, Tongji Medical College, Huazhong University of Science and Technology, Wuhan, China Full list of author information is available at the end of the article
}

and spiral artery lumen, rebuilding spiral artery [2]. The hypofunction of EVT invasion is the essential pathologic base of miscarriage, premature birth, stillbirth, fetus developmental retardation, gestational hypertension and other complications of pregnancy[3]. As being one of the symbolic proteins of EVT, c-erbB-2 protein participates in the process of EVT invasion[4]. In addition, Matrix metalloproteinase (MMP) is one of the key enzymes which affect EVT migration and invasion function, especially MMP-2 and MMP-9. At present, there are only a few studies on HCMV's influence on EVT invasion function. In order to explore the correlation of EVT invasion dysfunction and HCMV intrauterine infection which causes miscarriage, stillbirth and fetus developmental retardation, this paper adopts early pregnancy villous explant cultured in vitro, so as to observe HCMV's influence on EVT invasion function in villus. Here is the report:

\section{() Biomed Central}

(C) 2011 Tao et al; licensee BioMed Central Ltd. This is an Open Access article distributed under the terms of the Creative Commons Attribution License (http://creativecommons.org/licenses/by/2.0), which permits unrestricted use, distribution, and reproduction in any medium, provided the original work is properly cited. 


\section{Materials and methods Materials \\ Specimen and sources of the virus}

The tissue of villus is from healthy pregnant women whose peripheral blood is HCMV antibody negative and whose gestational age are between 5 to 10 weeks, who are voluntarily conducted abortion because of planned parenthood, from department of gynecology and obstetrics of Tongji Hospital of Tongji Medical College of Huazhong University of Science and Technology from March 2010 to July 2010. The research procedure complies with the ethnical standard drew by Ethics Committee of Tongji Hospital of Tongji Medical College. HCMV AD169 strain is provided by Hubei Province Institute of Viruses, while TCID $_{50}$ is $10^{-5}$.

\section{Sources of main reagents}

DMEM/Ham's F 12 culture medium (Gibco), standard fetal bovine serum (Hyclone), rabbit anti HCMV pp65 polyclonal antibody (Santa), rabbit anti c-erbB-2 polyclonal antibody, rabbit anti MMP-2 and MMP-9 polyclonal antibodies (Wuhan Boster Bio-engineering Co., Ltd), SP immunohistochemistry kit (Zhongshan Goldenbridge Biotechnology Co., Ltd), total protein extraction kit (BestBio), $\beta$-actin loading control antibody (Beijing Biosynthesis Biotechnology Co., Ltd) and so on.

\section{Methods}

\section{Villous explant culture and experimental grouping}

Improvements are made by referring to Dong cultural method[5]. The specific method is as follow: villous tissue was rapidly taken to laboratory by being placed in sterile pre-cooling D-hank's solution (containing $100 \mathrm{UI} / \mathrm{ml}$ penicillin and $100 \mu \mathrm{g} / \mathrm{ml}$ streptomycin), which were washed for three times for removing gore and non-cruor; it was put into serum-free DMEM/Ham's F 12 culture medium, and the apical tissue of villus (wet weight $40 \sim 50 \mathrm{mg}$ ) was carefully cut and inoculated in 24-hole cell culture plate, being cultured overnight in DMEM/Ham's F 12 culture medium containing $10 \%$ fetal bovine serum with $5 \% \mathrm{CO}_{2}$ at $37^{\circ} \mathrm{C}$; after throwing away the supernatant, it was changed to be DMEM/Ham's F 12 culture medium containing $3 \%$ fetal bovine serum. In infection group, 1 ul HCMV virus solution was added in every hole, while equal amount of PBS was added into every hole in control group; culture medium was absorbed, threw away in the two groups above after $2 \mathrm{~h}$ culture and washed by PBS for twice and added into complete medium; after $48 \mathrm{~h}$, villous explants were taken out. Total protein was extracted from part of villous tissue for Western blot detection. The rest of villous tissue was fixed for $24 \mathrm{~h}$ in formalin buffer solution and paraffin wax embedding sliced (thickness of slice was 3 um), for conducting pathological and immunohistochemical detection.

\section{Hematoxylin eosin(HE) staining}

Two groups of villous slice are being HE stained. Optical microscope is utilized to observe villus pathological changes.

\section{Immunohistochemistry staining}

Two groups of slices are taken to conduct conventional treatment. Immunohistochemistry streptavidin-perosidase (SP) method is used to detect HCMV pp65, c-erbB-2, MMP-2 and MMP-9, and the method please refers to reference[6]. Paraffin embedded sections were prepared in a microtome and de-paraffinized in a series of ethanols and xylenes. Sections were blocked with methanol containing $3 \% \mathrm{H}_{2} \mathrm{O}_{2}$, sequentially $10 \%$ goat normal serum, and incubated with rabbit anti HCMV pp65 polyclonal antibody, rabbit anti c-erbB-2 polyclonal antibody, rabbit anti MMP-2 and MMP-9 polyclonal antibodies (1:100 dilution) overnight at $4^{\circ} \mathrm{C}$. They were then sequentially treated with a biotinylated goat anti-rabbit antibody and a horseradish peroxidase(HRP) labelled streptoavidin for $1 \mathrm{~h}$ at $37^{\circ} \mathrm{C}$ respectively. Development was performed by treating the sections with a Liquid DAB-Plus Substrate kit. After counter-staining with haematoxylin, immunostaining of these proteins on the tissue sections was detected by light microscope. Under optical microscope, positive signals were nankeen or brown grains. HMIAS-2000 high definition color medical image analysis system was adopted to process images. Under high magnification, 10 visual fields were randomly observed, testing positive expression cells' average optical density for semi-quantitative statistical analysis.

Controls The above immunohistochemical procedures controls were performed replacing the primary antibody by PBS. Further controls were performed omitting the secondary antibody. The controls were always negative.

\section{Western Blot detection}

It is to detect c-erbB-2, MMP-2 and MMP-9 expression levels. Referring to the instruction of kits, two groups of villous total protein were extracted and stored in fridge at $-70^{\circ} \mathrm{C}$. Bradford method was used to measure protein concentration. In every sample, $50 \mu \mathrm{g} 10 \%$ SDS-PAGE was taken and wet transferred to PVDF membrane. 5\% skimmed milk powder was sealed for $2 \mathrm{~h}$ at room temperature. Rabbit anti c-erbB-2, MMP-2 and MMP-9 polyclonal antibodies were respectively added, cultured overnight at $4^{\circ} \mathrm{C}$. After TBST washing membrane, it was added into second antibody and cultured for $2 \mathrm{~h}$ at room temperature. TBST washes membrane and ECL reagent developed color. Exposure imaging was done under gel imager. Quantityone gel image analysis software detected different groups' protein and their loading control $\beta$-actin protein's absorbance value, showing target protein level by the ratio of target strip and average absorbance of loading control protein. 


\section{Statistical analysis}

These experiments above were repeated 3 times. SPSS 13.0 statistical software is adopted. Value of number is expressed by the mean \pm standard deviation. Differences among groups are conducted independent sample t testing, while $\mathrm{P}<0.05$ means the difference is of statistical significance.

\section{Results}

\section{Villus HE staining}

In control group, ST and CT were visible on villous surface, and cell boundary was clear. Villous interstitial substance was loose primitive interstitial substance. In interstitial substance, small blood vessels can be seen (Figure 1A). There was no obvious abnormality in villus of infected group(Figure 1B).

\section{Villus HCMV detection results}

Immunohistochemistry staining results were shown in Figure 2. When infected group was added into HCMV and cultured for $48 \mathrm{~h}, \mathrm{HCMVpp} 65$ signals showed almost all CT, EVT, ST, interstitial cells and around villous interstitial substance small blood vessels in villus (Figure 2B), while there was no HCMVpp65 antigen signal in control group(Figure 2A). HCMV was added into early pregnancy villus in vitro culture, after $48 \mathrm{~h}$, which also can cause villus infection. Virus can reproduce in CT, EVT, ST, interstitial cells and around villous interstitial substance small blood vessels in villus.

\section{c-erbB-2 protein expression level}

Immunohistochemistry staining results were shown in Figure $3 \mathrm{~A}, \mathrm{~B}, \mathrm{C}$, it was found that villous c-erbB-2 protein of control group and infected group expressed in a large quantity in ST remote plasma membrane, EVT, especially villous stem cell column remote EVT membrane, while expressed little in ST proximal end membrane and interstitial cells, and the expression level of infected group reduced $(\mathrm{P}<0.01)$. Western blot method detects the two groups'villus(Figure 3D, E), and c-erbB-2 protein aimed strips can be detected in $185 \mathrm{kDa}$, where
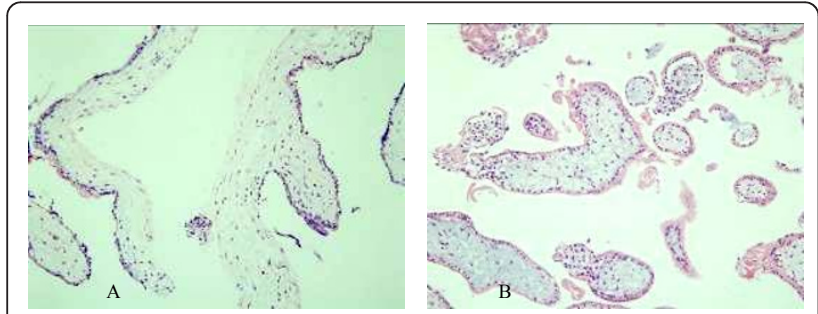

Figure 1 Histology of two groups'villous explants under $\mathrm{HE}$ staining. A. Human villous explants in vitro HE $\times 200$; B. Human villous explants infected by HCMV in vitro HE $\times 200$.

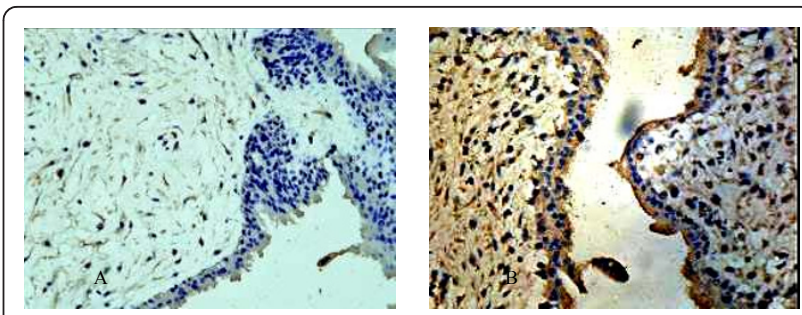

Figure 2 The expression of HCMVpp65 antigen in two groups'villous explants detected by immunohistostaining. A. The expression of HCMVpp65 antigen in normal human villous explants detected by immunohistostaining $\times 400$; $B$. The expression of HCMVpp65 antigen in human villous explants infected by HCMV detected by immunohistostaining $\times 400$.

infection group's expression level decreased $(\mathrm{P}<0.05)$. The results above indicate that infected HCMV may lead to the decrease of villous c-erbB-2 protein expression.

\section{MMP-2 and MMP-9 protein expression level}

Immunohistochemistry staining showed that the two groups' MMP-2 proteins both expressed in EVT endochylema in abundance, but little in VT, ST and interstitial substance(Figure 4A, B); MMP-9 protein expresseed largely in villous interstitial substance, EVT and VT endochylema and little in ST(Figure 5A, B). The expression level of MMP-2 and MMP-9 of infected group both decreased $(\mathrm{P}<0.01$ and 0.05 , the results are shown respectively in Figure 4C and Figure 5C); when Western blot method was used, target strips can be detected at $72 \mathrm{kDa}$ and $92 \mathrm{kD}$ in the two groups(Figure 4D and Figure 5D), but the two protein expression levels both reduced in infected group $(\mathrm{P}<0.05$, the results are shown respectively in Figure $4 \mathrm{E}$ and Figure $5 \mathrm{E}$ ). The results above indicate that infected HCMV may lead to the decrease of villous MMP-2 and MMP-9 protein expression.

\section{Discussion}

HCMV is the most common pathogen in intrauterine infection. Among the new born babies in Europe and America, HCMV infection rate is $2 \%$, while that in developing countries is even higher[7]. HCMV intrauterine infection may cause miscarriage, stillbirth, fetus developmental retardation, central nervous system damage and other serious results, but its specific pathogenesis remains unknown. HCMVpp65 protein is the major envelope protein in early stage of HCMV reproduction. The definite symbol of HCMV active infection is controlling HCMV gene expression, inhibiting host's cell metabolism and promoting replication of virus[8].

In the later stage of blastula implantation, $\mathrm{CT}$ is divided into VT and EVT. VT locates in inner layer of 


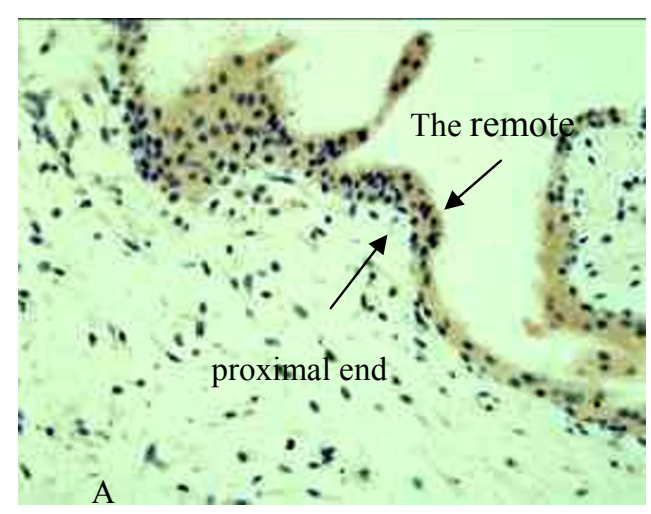

The effect on c-erbB-2 of human villous explants infected by HCMV

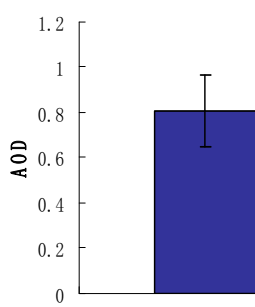

CTR

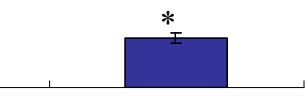

HCMV Groups

C

*Compared with control group, the expression of c-erbB-2 protein in HCMV group decreased significant $1 \mathrm{y}, \mathrm{P}<0.01$

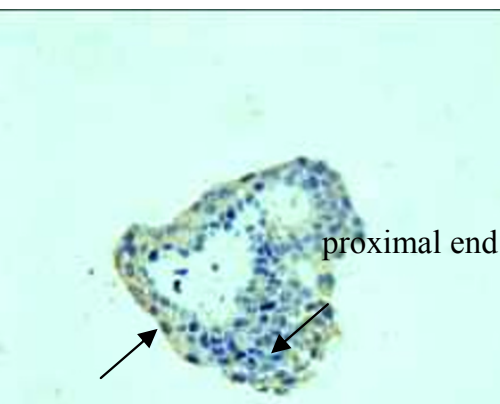

The remote

B

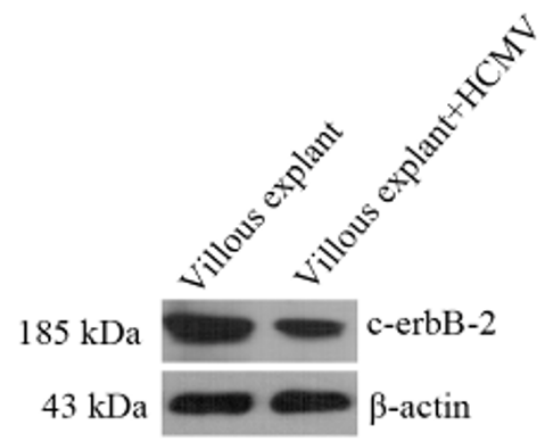

D

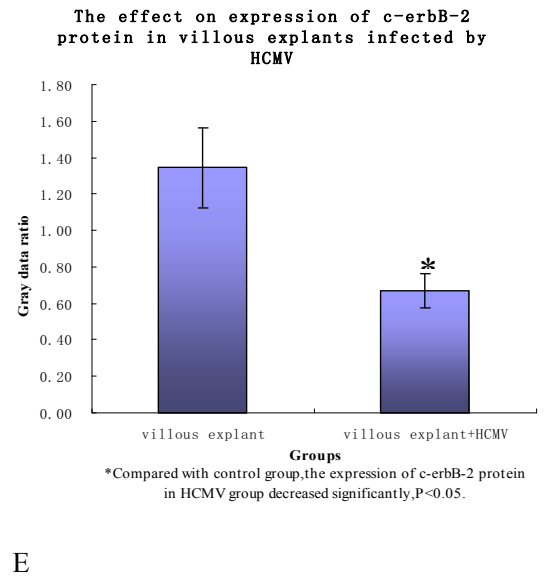

Figure 3 The expression of c-erbB-2 protein in two groups' villous explants. A, B. In the villus of two groups, c-erbB-2 protein mainly expressed in ST remote membrane, EVT especially villous stem cell column remote EVT membrane, but little in ST proximal end membrane and interstitial cells; C. According to the comparison of average optical density of two groups' c-erbB-2 protein expression, the protein expression of infected group reduced obviously $(P<0.01)$; D. Western blot method was used to detect c-erbB-2 protein of two groups' villus and the protein was detected at 185kDa; E. In infected group, $c$-erbB-2 protein expression level reduced $(p<0.05)$. Above these experiments were repeated 3 times. 

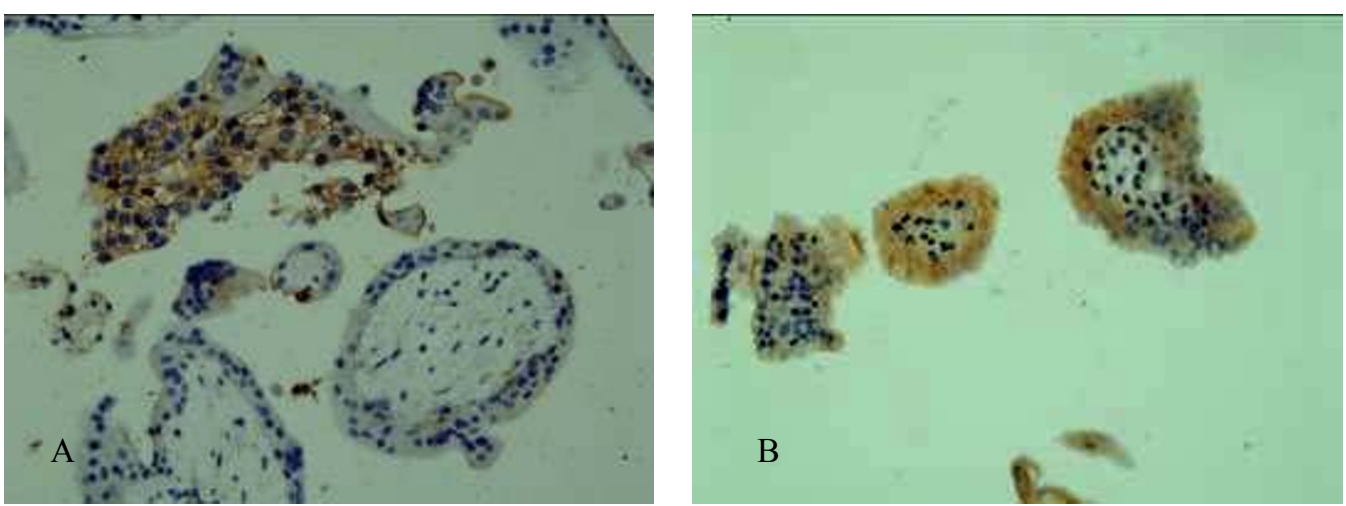

The effect on MMP- 2 of human

villous explants infected by $\mathrm{HCMV}$

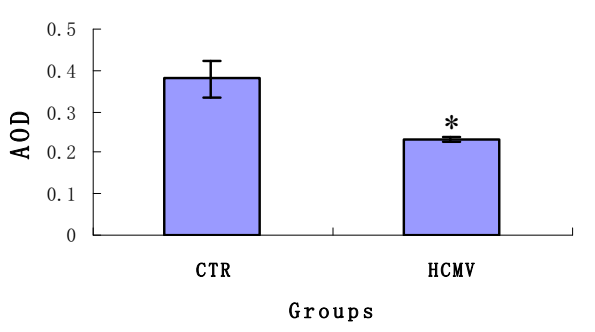

*Compared with control group, the expression of MMP-2 protein in HCMV group decreased

$\mathrm{C}$ significantly, $\mathrm{P}<0.01$.

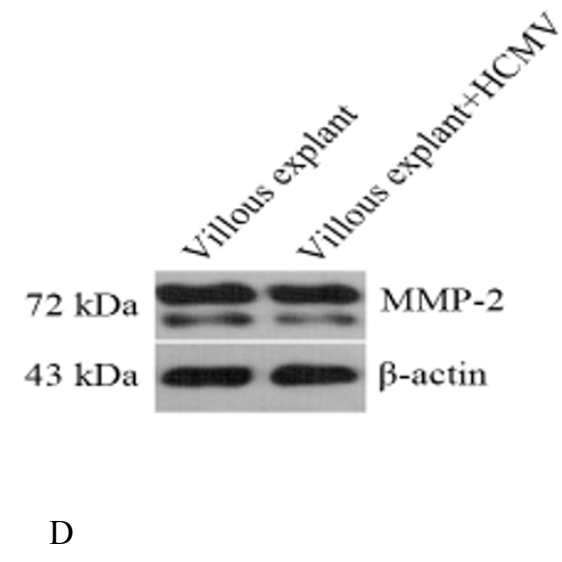

The effect on expression of MMP-2 protein in villous explants infected by HCMV

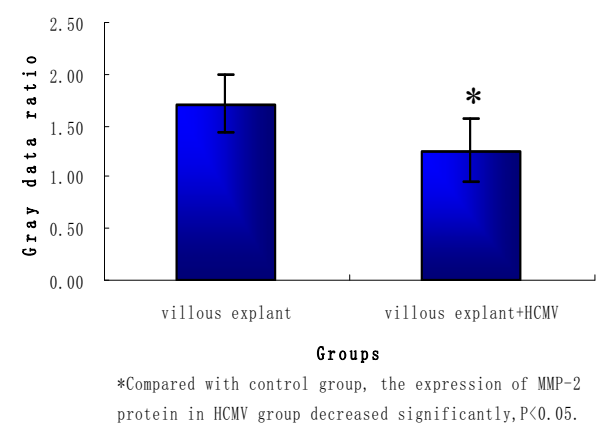

Figure 4 The expression of MMP-2 protein in two groups' villous explants. A, B. In the villus of two groups, MMP-2 protein expressed mainly in EVT endochylema and little in VT, ST and interstitial substance; C. According to the comparison of average optical density of two groups' MMP-2 protein expression, the protein expression significantly decreased in infected group $(P<0.01)$; $D$. Western blot method was used to detect MMP-2 protein of two groups' villus and the protein was detected at 72kDa; E. In infected group, MMP-2 protein expression level reduced $(P<0.05)$. Above these experiments were repeated 3 times.

villus, being active in karyokinesis and able to fuse and form ST which floats on the surface of villus, functioning as internal secretion, immunity and material exchange; EVT proliferates and migrates, gathering together to be cell column and invading into uterus decidua and superficial muscle layer, forming anchoring villus, which enables placenta to be fixed in vascular system of uterus[9]. During embryo implantation, EVT invades decidua and uterus superficial muscle layer and physiologically remodels uterine spiral arteries, which is 

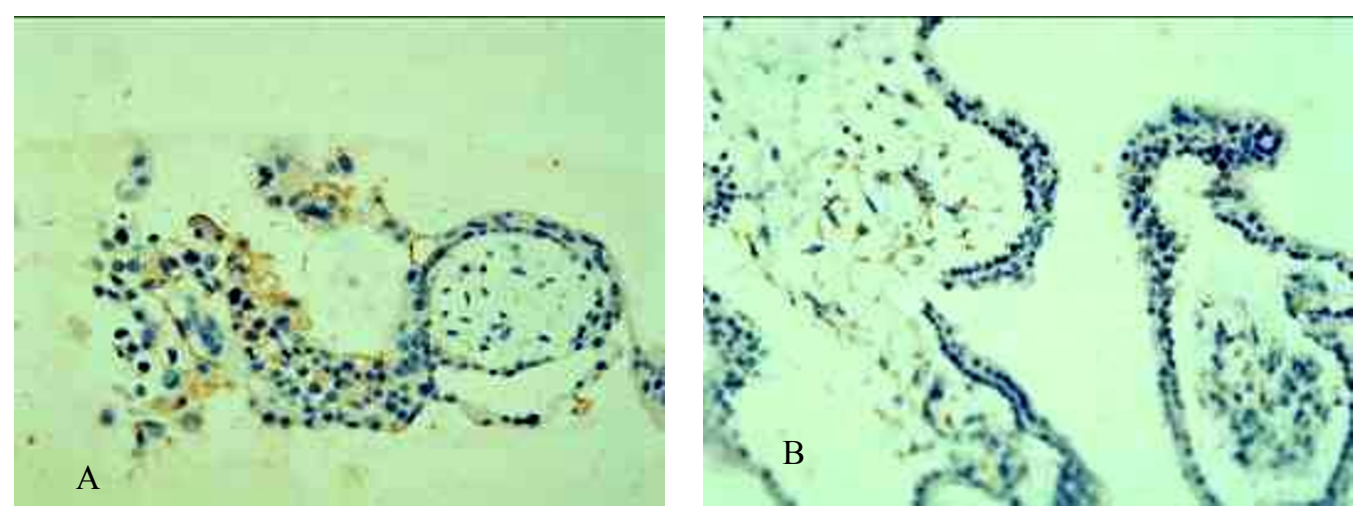

The effect on MMP-9 of human villous explants infected by HCM
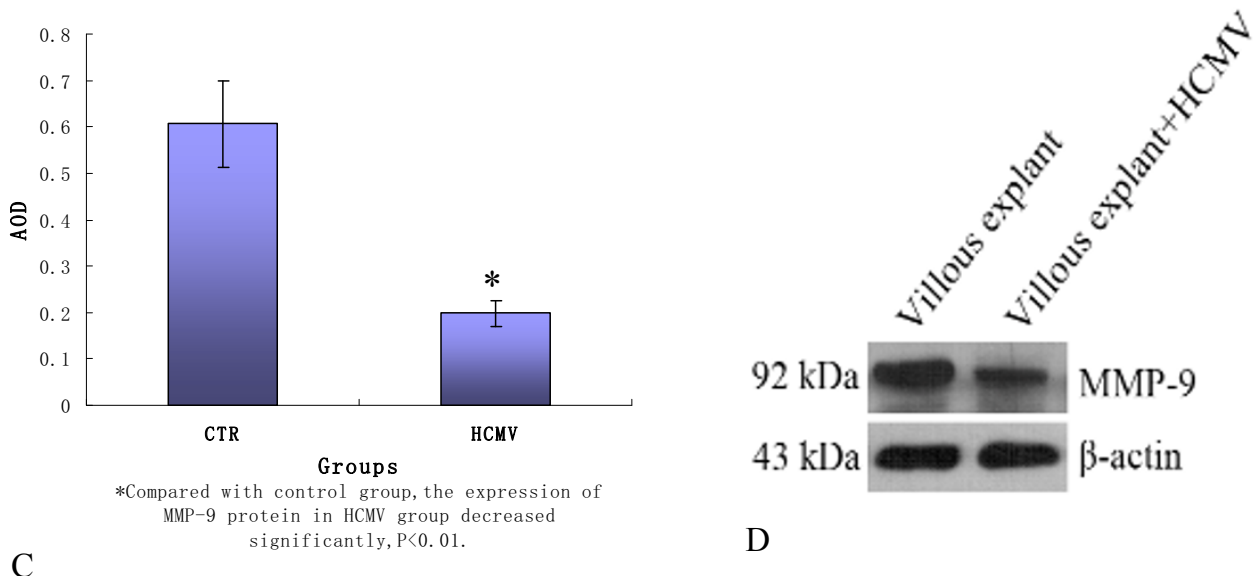

C

The effect on expression of Mup-9 protein in villous explants infected by HCMV

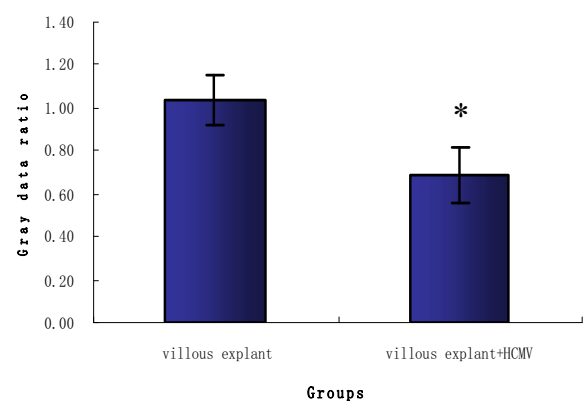

E HCWV group decreased significantly, $\mathrm{P}<0.05$.

Figure 5 The expression of MMP-9 protein in two groups' villous explants. A, B. In the villus of two groups, MMP-9 protein expressed mainly in villous interstitial substance, EVT and VT endochylema but little in ST; C. According to the comparison of average optical density of two groups' MMP-9 protein expression, the protein expression significantly decreased in infected group $(P<0.05)$; D. Western blot method was detected MMP-9 protein of two groups' villus and the protein was detected at 92kDa; E. In infected group, MMP-9 protein expression level reduced $(P<0.05)$. These experiments above were repeated 3 times. 
the necessary condition of successful pregnancy. EVT invasion function decreases, which may lead to miscarriage, stillbirth, fetus developmental retardation, gestational hypertension and other complications of pregnancy. In order to probe the molecular mechanism of HCMV intrauterine infection's causing miscarriage, stillbirth, fetus developmental and other abnormal outcomes, early-pregnancy villus of healthy women in normal pregnancy is used for culturing in vitro, and HCMV is added into culture medium. After $48 \mathrm{~h}$, it is observed that villus HCMV infection condition and the c-erbB-2, MMP-2 and MMP-9's expression levels in villus and characteristics of space distribution change. Immunohistochemistry staining results show that HCMVpp65 antigen signal does not show in control group, but in almost all CT, EVT, ST, interstitial cells and around small blood vessels of villous interstitial substance, that is, after adding HCMV into villous culture medium, 48 $\mathrm{h}$ later, villous cells are infected.

c-erbB-2 is part of epidermal growth factor receptor, being a kind of oncogene protein with tyrosine kinase activity and closely linked with embryogenesis, tissue repair and regeneration. It is found that c-erbB-2 highly expresses in EVT which is cultured in vitro, being one of the symbolic proteins of EVT[10-12]. c-erbB-2's expression time and space is highly consistent with EVT invasion function in villus. Therefore, c-erbB-2 can be used to analyze EVT invasion function in villus. This experimental result indicates that c-erbB-2 mainly expresses in early-pregnancy ST remote plasma membrane, EVT, especially villous stem cell column remote EVT's plasma membrane, being consistent with the reports of Mühlhauser et al[13]; HCMV infection brings about the reduce of villous c-erbB-2 expression level, which probably is related with the decrease of EVT invasion function.

MMP is one of the key enzymes which affect EVT migration and invasion function, being characteristic of specificity and timeliness in various trophocytes. Maternal-fetal interface's physical environment, changes in extracellular matrix components, growth factor, cell factor and hormone together maintain the balance of MMP/tissue inhibitor of metalloproteinase(TIMP) system and control the cytotrophoblast cells' proliferation, differentiation and invasion. It is found in study that MMP-2 and MMP-9 are the main stroma metalloprotease which mediate EVT invasion[14]. MMP-9 highly expresses in early and medium pregnancy EVT; MMP-2 expresses in all EVT during the whole gestational period and is active in early and medium pregnancy. The changes of expression level and activity are the same as EVT invasion function's changes[15]. In our study, villous immunohistochemistry staining shows that MMP-2 and MMP-9 protein express largely in early pregnancy villous EVT endochylema, while MMP-9 protein also expresses in high level in early pregnancy villous interstitial substance and VT endochylema; HCMV infection results that villous MMP-2 and MMP-9 expression level both significantly decrease. Villous MMP-2 and MMP-9 expression level reduces, which will lower EVT's proliferation, differentiation and invasion function. The mechanism of HCMV damaging the balance of MMP/ TIMP system still remains unclear.

\section{Conclusions}

This paper tentatively proves with in vitro experiments that HCMV can infect almost all CT, EVT, ST, interstitial cells and villous interstitial substance inside villus in vitro; HCMV reproduces and proliferates in early pregnancy villus, arousing villous c-erbB-2, MMP-2 and MMP-9 protein expression level decrease, which may be one of the pathogenesis that HCMV infection causes the reduce of EVT invasion function, miscarriage, stillbirth, cerebellar malformation, fetus developmental retardation and other offspring abnormalities. Next, related researches will be conducted through whole animal experiments.

\section{List of abbreviations}

HCMV: Human cytomegalovirus; CT: cytotrophoblast; VT: villous trophoblast; EVT: extravillous cytotrophoblas; ST: syncytiotrophoblast; MMP: matrix metalloproteinase; TIMP: tissue inhibitor of metalloproteinase; HE: hematoxylin eosin.

\section{Acknowledgements}

The research in the authors' laboratories has been funded by the National Natural Science Foundation of China (Grant NO30672243). Special thanks to our present and former lab members for their contributions over the years.

\section{Author details}

'Department of Obsterics and Gynecology, Tongji Hospital, Tongji Medical College, Huazhong University of Science and Technology, Wuhan, China. ${ }^{2}$ Department of Obsterics and Gynecology, Tai'an City Central Hospital, Tai'an, China.

\section{Authors' contributions}

LT performed all experiments and drafted the manuscript. CJ, YZZ, XJ and ZDD participated in the design of the study and contributed to drafting the manuscript. CSH conceived of the study, and participated in its design and coordination and revised the manuscript. All authors read and approved the final manuscript.

\section{Competing interests}

The authors declare that they have no competing interests.

Received: 10 January 2011 Accepted: 11 March 2011

Published: 11 March 2011

\section{References}

1. Hyde TB, Schmid DS, Cannon MJ: Cytomegalovirus seroconversion rates and risk factors: implications for congenital CMV. Rev Med Virol 2010, 20:311-26.

2. Takako Yamamoto-Tabata, Susan McDonagh, Hsin-Ti Chang, et al: Human Cytomegalovirus Interleukin-10 Downregulates Metalloproteinase Activity and Impairs Endothelial Cell Migration and Placental Cytotrophoblast Invasiveness In Vitro. J Virol 2004, 78:2831-2840. 
3. Lorenzi T, Marzioni D, Giannubilo S, et al: Expression patterns of two serine protease $\mathrm{HtrA} 1$ forms in human placentas complicated by preeclampsia with and without intrauterine growth restriction. Placenta 2009, 30:35-40.

4. Jokhi PP, King A, Loke YW: Reciprocal expression of epidermal growth factor receptor (EGF-R) and c-erbB2 by non-invasive and invasive human trophoblast populations. Cytokine 1994, 6:433-42.

5. Dong M, Ding G, Zhou J, et al: The effect of trophoblasts on $T$ lymphocytes: possible regulatory effector molecules-a proteomic analysis. Cell Physiol Biochem 2008, 21:463-72.

6. Lockwood CJ, Oner C, Uz YH, et al: Matrix metalloproteinase 9 (MMP9) expression in preeclamptic decidua and MMP9 induction by tumor necrosis factor alpha and interleukin 1 beta in human first trimester decidual cells. Biol Reprod 2008, 78:1064-72.

7. LaMarca $H L$, Nelson $A B$, Scandurro AB, et al: Human cytomegalovirusinduced inhibition of cytotrophoblast invasion in a first trimester extravillous cytotrophoblast cell line. Placenta 2006, 27:137-47.

8. Cristea IM, Moorman NJ, Terhune SS, et al: Human cytomegalovirus pUL83 stimulates activity of the viral immediate-early promoter through its interaction with the cellular IFI16 protein. J Virol 2010, 84:7803-14.

9. Takako Yamamoto-Tabata, Susan McDonagh, Hsin-Ti Chang, et al: Human Cytomegalovirus Interleukin-10 Downregulates Metalloproteinase Activity and Impairs Endothelial Cell Migration and Placental Cytotrophoblast Invasiveness In Vitro. J Virol 2004, 78:2831-2840.

10. Tarrade A, Lai Kuen R, Malassiné A, et al: Characterization of human villous and extravillous trophoblasts isolated from first trimester placenta. Lab Invest 2001, 81:1199-211.

11. Oki N, Matsuo H, Nakago S, et al: Effects of 3,5,3'-triiodothyronine on the invasive potential and the expression of integrins and matrix metalloproteinases in cultured early placental extravillous trophoblasts. $J$ Clin Endocrinol Metab 2004, 89:5213-21.

12. Kliman HJ: Uteroplacental blood flow: the story of ecidualization, menstuation and trophoblast invasion. Am J Pathol 2000, 157:1759-1768.

13. Mühlhauser J, Crescimanno C, Kaufmann P, et al: Differentiation and proliferation patterns in human trophoblast revealed by c-erbB-2 oncogene product and EGF-R. J Histochem Cytochem 1993, 41:165-73.

14. Ioannidis I, Dimo B, Karameris A, et al: Comparative study of the immunohistochemical expression of metalloproteinases 2, 7 and 9 between clearly invasive carcinomas and "in situ" trophoblast invasion. Neoplasma 2010, 57:20-8.

15. Isaka K, Usuda S, Ito H, et al: Expression and activity of matrix metalloproteinase 2 and 9 in human trophoblasts. Placenta 2003, 24:53-64.

\section{Submit your next manuscript to BioMed Central and take full advantage of:}

- Convenient online submission

- Thorough peer review

- No space constraints or color figure charges

- Immediate publication on acceptance

- Inclusion in PubMed, CAS, Scopus and Google Scholar

- Research which is freely available for redistribution 